\title{
Linear cutaneous lupus erythematosus following the lines of Blaschko - Case report ${ }^{*}$
}

\author{
Ayana Karla de Oliveira Ferreira Marinho ${ }^{1,2}$ \\ Deborah Maria de Castro Barbosa ${ }^{4}$ \\ Daniela Mayumi Takano ${ }^{4}$
}

\author{
Ticiana Batista Ramos ${ }^{3}$ \\ Valter Kozmhinsky ${ }^{4}$ \\ Marcella Maria de Souza Araújo Figueira ${ }^{4}$
}

DOI: http:/ / dx.doi.org/10.1590/abd1806-4841.20163800

\begin{abstract}
Chronic cutaneous lupus erythematosus in a linear configuration is rare, particularly in children, demonstrating similar incidence in both genders, no photo-sensitivity and lower probability of progression to systemic disease. We describe the case of a 9-year-old girl who presented erythematous papules with central atrophy on the upper and lower right limbs, asymptomatic and following the lines of Blaschko, since age four. Histological examination showed atrophy of the epidermis with aggression from epidermal-dermal interface and periadnexal and perivascular lymphocytic inflammatory infiltrate. Laboratory tests showed ANA in a titer of 1:320, in a dense and fine speckled pattern. Due to the rarity of presentation and location of the disease, this case is reported here.
\end{abstract}

Keywords: Lupus erythematosus, cutaneous; Lupus erythematosus, discoid; Lupus erythematosus, systemic; Mosaicism

\section{INTRODUCTION}

Cutaneous lupus erythematosus in a linear configuration is rare, especially in children. Less than $2 \%$ of cases appear before age $10 .{ }^{1}$ It affects equally both genders, shows no photosensitivity and less probability of evolution to systemic disease. ${ }^{2}$ The term linear cutaneous lupus erythematosus (LCLE) is currently employed since clinical lesions lack the classic discoid shape. ${ }^{3}$

Such linear configuration follows the lines of Blaschko, which were described for the first time in 1901 by Alfred Blaschko, who reported the presence of several linear diseases with characteristic disposition. ${ }^{4}$
We describe a 9-year-old patient with, papular and erythematous lesions with central atrophy on the upper and lower right limbs, asymptomatic, and following the lines of Blaschko. We highlight the unusual clinical presentation of this disorder.

\section{CASE REPORT}

Female patient, 9-year-old, phototype IV, presented a history of onset of papular and erythematous cutaneous lesions, some violaceous, with central atrophy and asymptomatic, since age 4 . Such lesions had a linear appearance, following the lines of Blaschko, on the upper and lower right limbs (Figures 1 - 4). There were

Received on 26.06.2014

Approved by the Advisory Board and accepted for publication on 15.12.2014

* Work performed at Instituto de Medicina Integral Professor Fernando Figueira (Imip) - Recife (PE), Brazil. Financial Support: None.

Conflict of Interest: None.

Grupo Universitário Maurício de Nassau (Uninassau) - Recife (PE), Brazil.

Real Hospital Português de Beneficência de Pernambuco - Recife (PE), Brazil.

Escola Multicampi de Ciências Médicas (UFRN) - Caicó (RN), Brazil.

Instituto de Medicina Integral Professor Fernando Figueira (Imip) - Recife (PE), Brazil. 


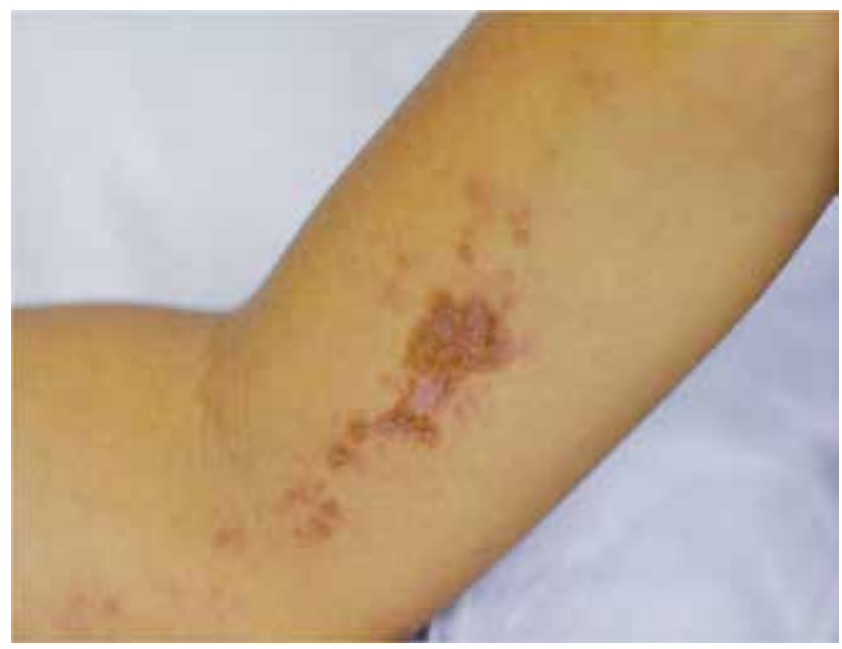

FIGURE 1: Erythematous brownish papules with central atrophy, following the lines of Blaschko, on the upper right limb

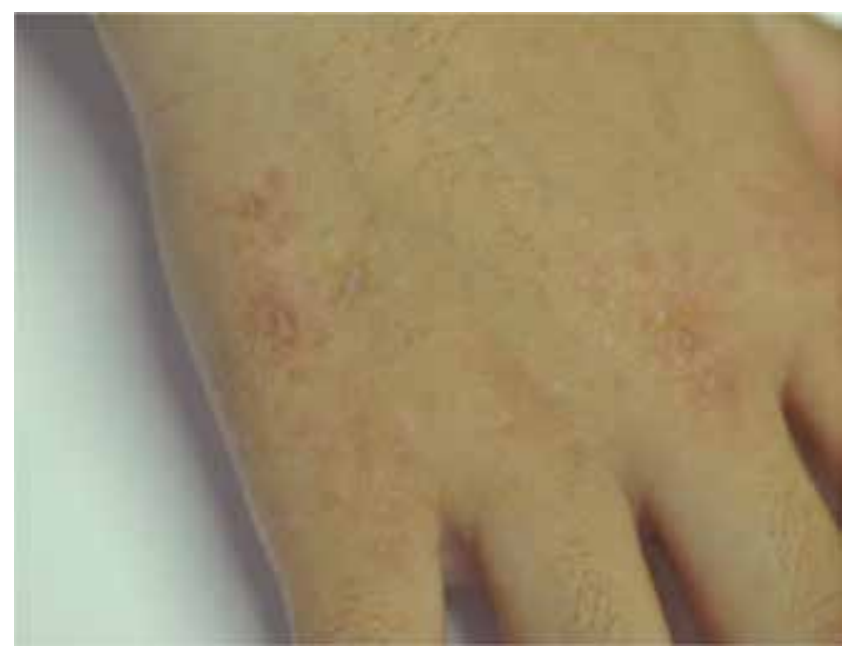

FIGURE 2: Erythematous papules on the back of right hand no reports of similar cases in the family or history of trauma or photosensitivity.

A biopsy revealed atrophy of the epidermis, with aggression of epidermal-dermal interface and periadnexal and perivascular lymphocytic inflammatory infiltrate (Figure 5). Additionally, PAS staining showed thickening and irregularities of the basement membrane

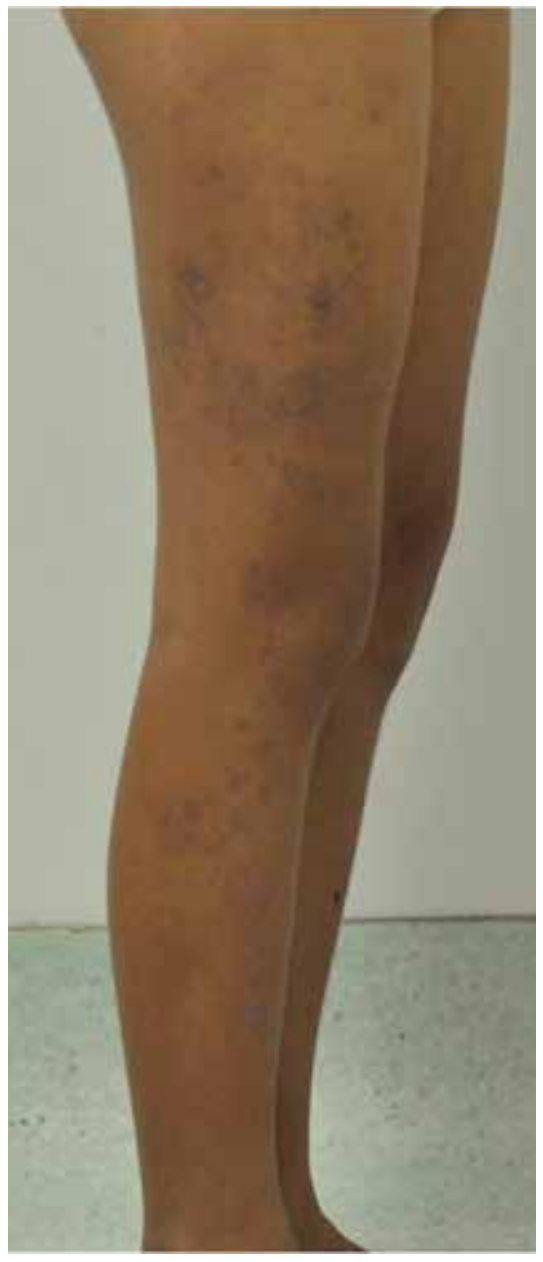

FiguRE 3:

Erythematous purplish papules with central atrophy, following the lines of Blaschko, on the lower right limb

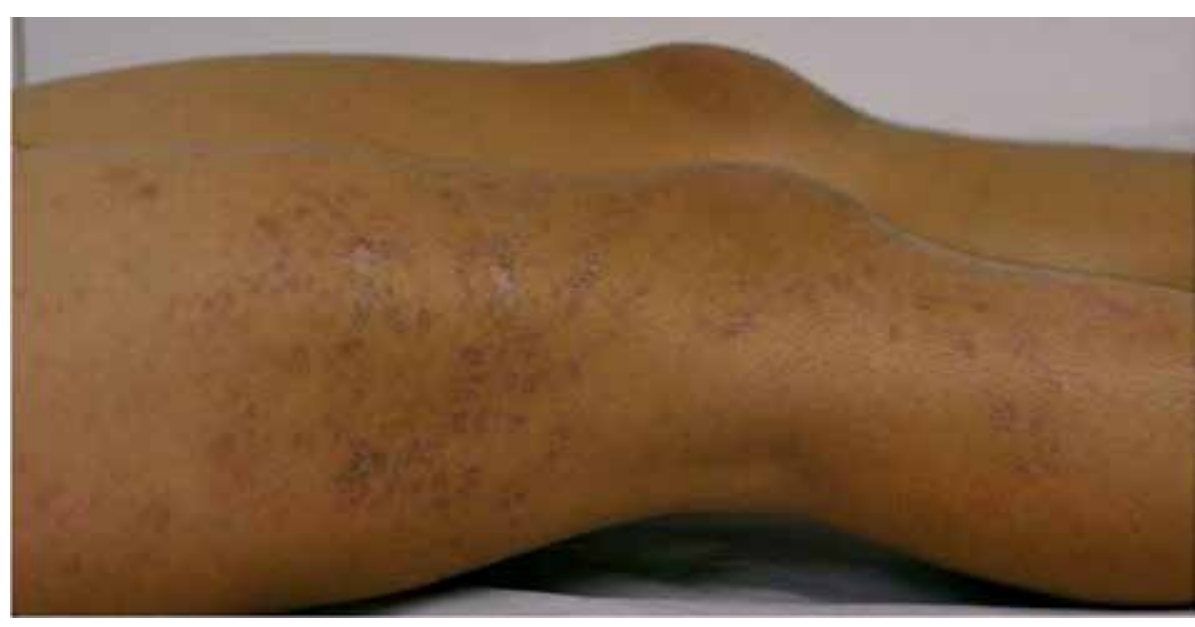

FIGURE 4:

Erythematous purplish papules presenting central atrophy, on the side of right thigh 


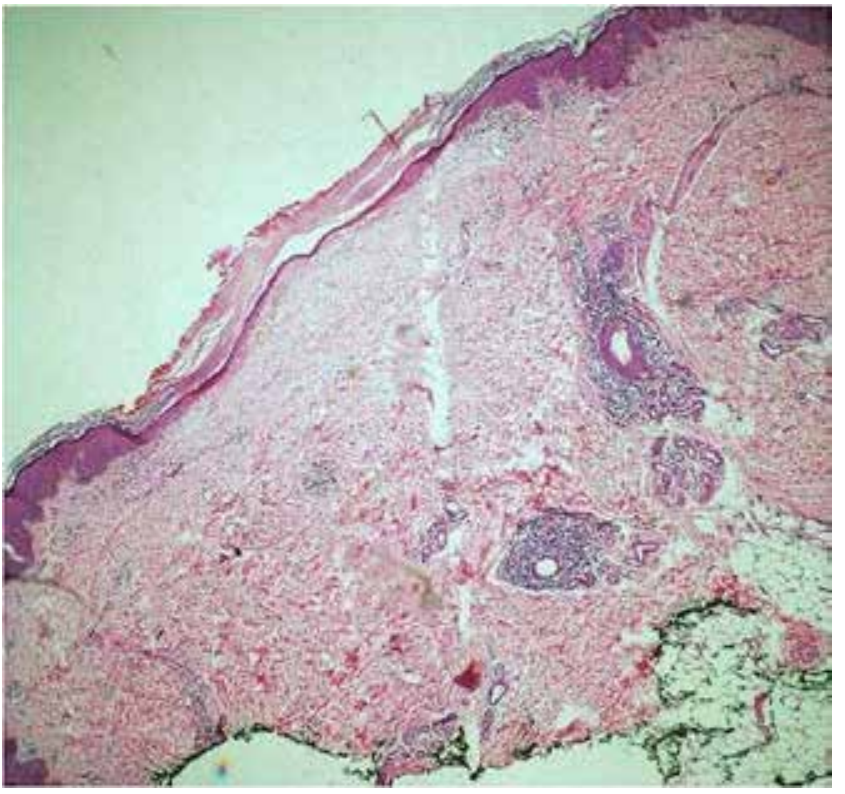

FiguRe 5: Epidermal atrophy, perivascular and periadnexal dermal inflammatory infiltrate, and vacuolization of basal layer (aggression of epidermal-dermal interface) (HE, 40x)

(Figure 6). ANA was postive (1:320, dense fine speckled pattern) and other laboratory test did not show systemic involvement.

Treatment with hydroxychloroquine $(150 \mathrm{mg}$ / day) was instituted with gradual remission. No relapse was observed within a one-year follow-up.

\section{DISCUSSION}

Cutaneous lupus erythematosus has many clinical presentations, divided in acute, subacute and chronic. Its linear presentation is very uncommon (than 20 cases described), especially when the most involved site - the face - is not affected. ${ }^{5}$

The origin of the lines of Blaschko remains controversial, with two theories explaining the pathogenesis. According to the first one, these lines may be the result of a mosaicism of cutaneous cells, in which there would be two or more distinct cell populations, originated from a genetically homogeneous zygote, arising from a somatic mutation, functional disparity of the $\mathrm{X}$ chromosome or gonadal genetic mutation. The second theory proposes that the lines of Blaschko result from an isolated cutaneous segmentary growth. ${ }^{6}$

Several inflammatory dermatoses follow the lines of Blaschko or present linear configurations. It is necessary to perform a differential diagnosis between LCLE and striated lichen, psoriasis, morphea, lichen planus and especially lichen sclerosus and atrophic.?

The majority of patients described in the literature developed the disease during childhood, with an average age of 15.8 (ranging from 3 to 42 years) and an average of one year and a half between the onset of lesions and diagnosis. There were lesions that followed the lines of Blaschko in all patients, and they could be seen mainly on the face and neck, demonstrating the most typical presentation of the disease. The involvement of members and body was

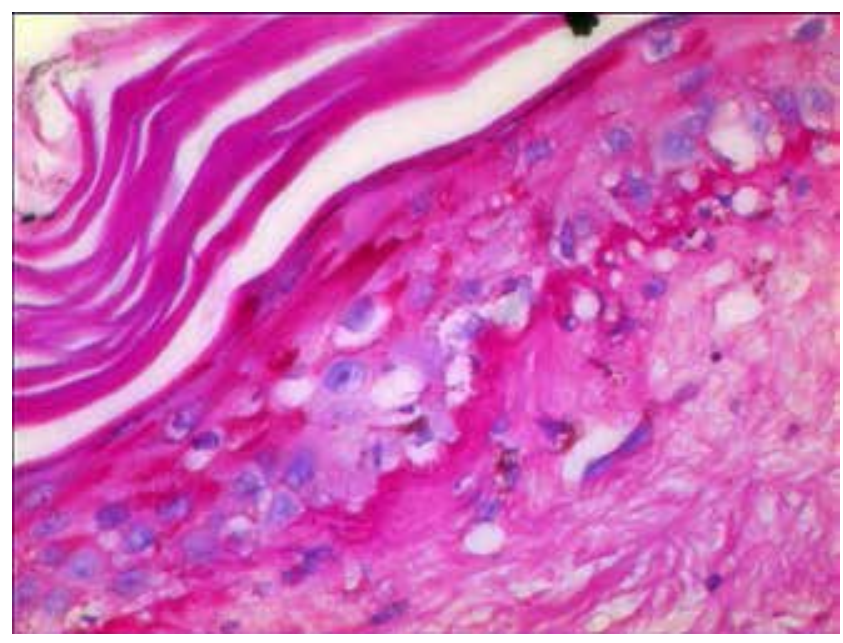

Figure 6: Basal layer thickening (PAS, 400x)

uncommon and, in the majority of cases, only a single anatomical site was affected. ${ }^{8}$

All published cases presented histological confirmation of lesions. In cases in which the histological diagnosis is unclear, direct immunofluorescence is recommended, in which granular deposits of IgM, IgG and IgA and complement in the dermo-epidermal junction zone and around dermal vessels can be observed. Such procedure is not mandatory for confirmation of the disease. ${ }^{9}$

LCLE in childhood presents less probability of evolving to systemic disease. In published studies, few patients presented positive ANA, in low titers (1:80), predominantly in homogeneous or speckled pattern. No systemic involvement has been reported. ${ }^{9}$

Hydroxy-chloroquine, a first-line drug, promotes remission of lesions in most cases along with a favorable safety profile. Dapsone, methotrexate, moderate to high potency topical corticosteroids and topical calcineurin inhibitors can also be employed in patients that are unresponsive to hydroxyxchloroquine or when this drug is contraindicated. ${ }^{10}$

The case presented shows the involvement of the limbs in two distinct anatomical sites, following the lines of Blaschko, in a child whose age group is compatible with described cases of LCLE. Additional investigations, based on clinical (such as photosensitivity and renal, hepatic, hematologic, neurological and inflammatory functions) and immunological criteria, yielded positive ANA results (1:320), a dilution higher than usual for such cases.

Linear lichen planus, or zosteriform, is the most important differential diagnosis. Similar histological findings in hematoxylin-eosin staining, such as liquefaction degeneration of the basal layer and the presence of lymphocytary infiltrate, were associated with features of LCLE such as epidermal atrophy, follicular plugging and 
perivascular and periadnexal infiltrate, infrequent hypergranuloisis and fewer colloid bodies. Thickening of the basal membrane could be demonstrated by PAS staining, providing the histological diagnosis of LCLE.

The prescribed treatment was adequate to inhibit the activity of the disease, and after its implementation there was gradual improvement of lesions. Additional medications were not introduced. The reported case demonstrates an atypical presentation of LCLE in view of its rarity, clinical picture and topography of lesions, reasons why describing it was considered important.]

\section{REFERENCES}

1. Requena C, Torrelo A, de Prada I, Zambrano A. Linear childhood cutaneous lupus erythematosus following blaschko lines. J Eur Acad Dermatol Venereol. 2002;16:618-20.

2. Lee MW, Choi JH, Sung KJ, Moon KC, Koh JK. Linear Cutaneous Lupus Erythematosus in the Lines of Blascko. Pediatr Dermatol. 2001;18:396-9.

3. Engelman D, Kotz E III, Maize J. Linear Cutaneous Lupus Erythematosus in the Lines of Blaschko. Pediatr Dermatol. 2007;24:125-9.

4. Seitz CS, Bröcker EB, Trautmann A. Linear variant of chronic cutaneous lupus erythematosus: a clue for the pathogenesis of chronic cutaneous lupus erythematosus? Lupus. 2008;17:1136-9.

5. Verma SB, Wollina U. Chronic disseminated discoid lupus erythematosus with linear lesions following Blaschko's lines on forearm and hand. J Dtsch Dermatol Ges. 2012;10:129-30.

6. Daldon PE, Lage R. Linear chronic discoid lupus erythematosus following the lines of Blaschko. An Bras Dermatol. 2011;86:553-6.

7. Röckmann H, Feller G, Schadendorf D, Goerdt S. Subacute cutaneous lupus erythematosus on the lines of Blaschko. Eur J Dermatol. 2006;16:302-6.

8. Sàbat M, Ribera M, Bielsa I, Mangas C, Fernández-Chico N, Ferrándiz C. Linear lupus erythematosus following the lines of Blaschko. J Eur Acad Dermatol Venereol. 2006;20:1005-6.

9. Gaitanis G, Nomikos K, Chaniotakis I, Stergiopoulou C, Zioga A, Bassukas I. Linear cutaneous lupus erythematosus: a subset of childhood cutaneous lupus erythematosus. Lupus. 2009;18:759-61.

10. Kawachi $Y$, Taguchi S, Fujisawa $Y$, Furuta J, Nakamura $Y$, Ishii $Y$, et al. Linear childhood discoid lupus erythematosus following the lines of Blascko: successfully treated with topical tacrolimus. Pediatr Dermatol. 2011;28:205-7.

\author{
MAILING ADDRESS: \\ Ayana Karla de Oliveira Ferreira Marinho \\ Rua dos Coelhos, 300 \\ Boa Vista \\ 50070-550 - Recife - PE \\ Brazil \\ E-mail: ayanakarla@hotmail.com
}

How to cite this article: Marinho AKOF, Ramos TB, Barbosa DMC, Kozmhinsky V, Takano DM, Figueira MMS. Linear cutaneous lupus erythematosus following the lines of Blaschko - Case report. An Bras Dermatol. 2016;91(4):510-3. 